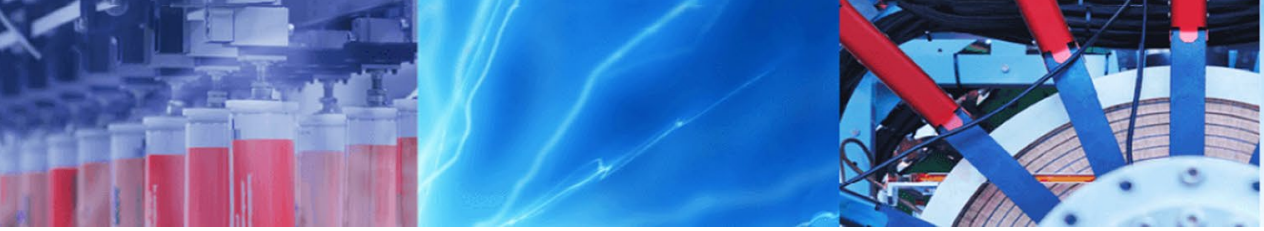

Research Article

\title{
Experimental and simulation analyses of the stress field of slider-on-block contact for static and slip states
}

\author{
Van Cuong Hoang ${ }^{1}$ (1) $\cdot$ Wanglong Zhan ${ }^{2} \cdot$ Yanfei Fang ${ }^{3} \cdot$ Ping Huang $^{1}$
}

Received: 23 October 2019 / Accepted: 15 January 2020 / Published online: 22 January 2020

(c) Springer Nature Switzerland AG 2020

\begin{abstract}
Contact problems have been widely investigated for many years. However, there are few reports on the real-time observation of shear stress evolution in friction process by experimental method. In this paper, the plane stress fields of slider-on-block contact in different contact states were observed by photoelasticity experiments and finite element simulation. In the static state, the slider is only subjected to a normal force by an L-shaped body above the slider while the block is fixed. However, in the slip state, the block is moved, and the slider is acted upon by normal and tangential forces. In both cases, the principal stress difference fields in the block obtained by photoelasticity and simulation were basically consistent. Additionally, based on simulation, the distributions of pressure and frictional stress on the contact surface were determined; the influences of the load and friction coefficient on the contact stress distributions were also investigated. Accordingly, some principles for contact stress distribution were obtained. The combination of experimental and simulation methods aims to be complementary, helping to better understand the nature of the contact stress field.
\end{abstract}

Keywords Photoelasticity · Finite element simulation · Principal stress difference · Pressure distribution · Friction

\section{Introduction}

The distributions of stress for different contact states are fundamental problems of contact mechanics. In general, the contact pressure and frictional stress on the contact surface and the maximum shear stress distribution in the subsurface are the main components mentioned. The distributions of pressure and frictional stress on the contact surface are often used as basic factors in calculating surface wear, while the maximum shear stress distribution is very important in predicting fatigue failure resulting in microcracking [1]. The study of these stress fields plays an important role in calculating the failure trends of machine parts and controlling the service life of mechanical elements.

In terms of theoretical research, first, the Hertz contact problem must be mentioned [2]. Based on a model of an elastic solid in contact, Hertz proposed the pressure $p$ of a cylinder in contact with a flat space as $p=p_{0}\left(1-(x / a)^{2}\right)^{-1 / 2}$, where $p_{0}$ is the maximum pressure and $a$ is half of the contact length. In fact, any friction surface always has roughness, when two surfaces are in contact, the real contact only occurs at dispersed points, which are often represented by basic contact models such as sphere-on-flat, cylinder-on-flat and cone-on-flat surfaces [3]. A series of studies about these basic models were proposed $[2,4-7]$. Basically, to facilitate the theoretical Electronic supplementary material The online version of this article (https://doi.org/10.1007/s42452-020-2061-4) contains
supplementary material, which is available to authorized users.

$\triangle$ Van Cuong Hoang, hoangcuong257@foxmail.com | 1 School of Mechanical and Automotive Engineering, South China University of Technology, Guangzhou 510640, China. ${ }^{2}$ Sino-German College of Intelligent Manufacturing, Shenzhen Technology University, Shenzhen 518118, Guangdong, China. ${ }^{3}$ State Key Laboratory of Tribology, Tsinghua University, Beijing 100084, China. 
calculation, the indenter part (sphere, cylinder or cone) is often assumed to be a rigid body, and the contact part is assumed to be an elastic half-space, or vice versa. In terms of these assumptions, these studies are mainly based on an available deformation of the contact region according to the boundary shape of the rigid part; then, elastic theory is used to calculate the pressure distribution. The study of frictional stress is mainly based on the assumption that the frictional stress is proportional to the contact pressure through the friction coefficient at the micro-level to determine the frictional stress distribution in cases of no slip, partial slip and slip [2,7]. The figures obtained for the fretting fatigue phenomenon of sphere-on-flat show that the frictional stress on the contact surface is uneven, causing stick and slip regions to appear. Regarding the study of the maximum shear stress distribution (or principal stress difference distribution) generated by the cylinder-on-flat contact model proposed by Huber in 1904 [2] and then developed by Hamilton [4], the theoretical results are consistent with the observed isochromatic of the photoelasticity experiment. Accordingly, the maximum shear stress does not immediately appear on the contact surface, that is, below the contact region, which indicates the risk of failure and microscopic cracking derived from inside the body [1]. Theoretical studies have shown a general picture of contact stress fields in simple models.

In the experimental research, some experimental methods were applied to measure the contact pressure distribution, such as a Tekscan pressure sensor [8] and a prescale measurement film $[9,10]$. However, these experimental methods must insert a thin film between the contacting bodies, which is a cause of measurement error. In addition, there are some limitations, such as that a prescale film can only determine the maximum contact pressure in the static state and that a Tekscan sensor cannot measure shearing stress.

According to the development of technical birefringent materials such as epoxy resin and polycarbonate, the photoelasticity experimental method has been applied to stress analysis. The phenomenon of stress-optics was discovered by Brewster in 1816 [11, 12], after which the law of stress-optics was determined by Maxwell in 1852 and then used to create an experimental method for analyzing the stress distribution inside a component part. In this way, it is possible to observe the principal stress difference field (isochromatic) and its direction (isoclinic) in an area of interest. Many studies have applied this method to contact stress analysis [13-17]. It appears that many techniques have been used to analyze the images obtained to find the isochromatic field and isoclinic field [17-22]; however, directly determining the stress distribution on the contact surface is still a difficult problem. Recently, Fang et al. [15] studied the application of photoelasticity combined with numerical simulation to stress analysis under elastohydrodynamic lubrication conditions. Hariprasad et al. [14] proposed fringe order distribution along the section beneath the contact surface of a flat punch with different friction coefficients. Zhan et al. [16] employed the photoelasticity method to observe stress fields in abrasive wear research. These studies mainly apply the photoelasticity method to observe the plane stress field beneath the contact region.

With the development of computers, the analysis of contact stress fields with the finite element simulation method helps us to obtain more intuitive results with high accuracy. Siswanto et al. [23] used the implicit and explicit finite element methods to study the cylinder-on-flat contact model using Abaqus software. The results of the contact pressure and the size of the contact area employed to compare with Hertzian contact theory showed very high coincidence. Bhushan and Peng [24, 25] studied twodimensional and three-dimensional multi-point contact based on the application of a random seeding algorithm to find the stress distribution on the contact surface. Masoudi Nejad et al. [26] employed the finite element method to predict fatigue crack growth in rolling contact under variable thermal loads, and the influence of loads and friction coefficients was also investigated.

As described above, the contact stress field of basic models has been investigated extensively for many years by different methods. For slider-on-block contact, which is also a basic contact model, although there are some theoretical studies [27], the results obtained for the stress field are still incomplete and not close to real contact conditions. In this article, photoelasticity experiments and finite element simulations were employed to survey the contact stress distributions of a two-dimensional slider-on-block contact model at different contact states. The principal stress difference fields were observed by photoelasticity and then were compared with simulation results. Based on simulation, some laws of the effects of contact states, loads and friction coefficients on the distributions of the principal stress difference, contact pressure and frictional stress were proposed and analyzed. The combination of experimental and simulation methods helped us to better understand the nature of the contact stress field.

\section{Theory}

\subsection{Physical model}

A slider sliding on a surface (or a block) is a model often used to describe the phenomenon of friction; this model appears in many classical friction problems and mechanical engineering, such as a wedge key (see Fig. 1a). In this 

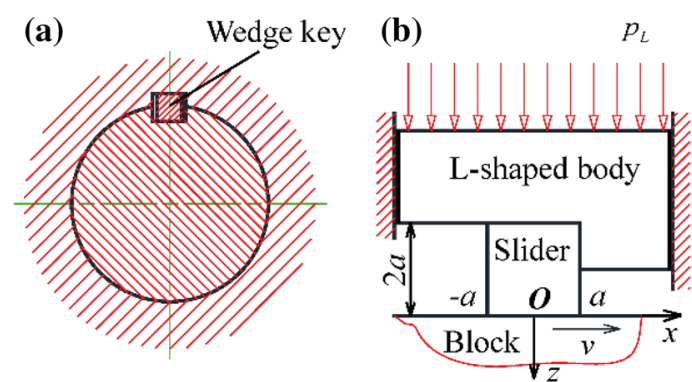

Fig. 1 Model of research a wedge key in assembly, b slider-onblock contact model

study, a slider in contact with a block is employed as the object of study.

A two-dimensional space (2D) model representing the object studied is shown in Fig. 1b. The slider is placed between the block and an L-shaped body. A uniform pressure distribution pressing on the top of the L-shaped body has a vertical moving trend, while the horizontal movement of the body is restricted. The L-shaped body presses the slider in contact with the block. The block is fixed in the static state and is moved from left to right in the slip state. In both the static and slip cases, we mainly illustrate the principal stress difference distribution (or maximum shear stress field) in the block and the distributions of the contact pressure and frictional stress on the contact region between the slider and block. In this model, the contact surfaces are assumed to be smooth with no roughness, and the materials are assumed to be elastic.

\subsection{Basic equations}

According to contact mechanics, when two elastic surfaces are in contact, the traction stresses (normal pressure $p(s)$ and tangential stress $q(s)$ ) and the deformation $\bar{u}_{z}$ on the contact surface are related as [2]:

$\bar{u}_{z}=-\frac{2}{\pi E^{*}} \int_{-b}^{a} p(s) \ln |x-s| d s-\frac{1}{2 G^{*}}\left(\int_{-b}^{x} q(s) d s-\int_{x}^{a} q(s) d s\right)+C$

Here, $E^{*}$ is the equivalent Young's modulus, $G^{*}$ is the equivalent shear modulus, $C$ is a constant, and $[-b, a]$ is the area of loading (see Fig. 2).

With the recognition of Coulomb's law at the microscopic level, the relationship between frictional stress $q(s)$ and normal pressure $p(s)$ on the contact surface is written as [27]:

$q(s)=\mu p(s)$

where $\mu$ is the coefficient of friction.

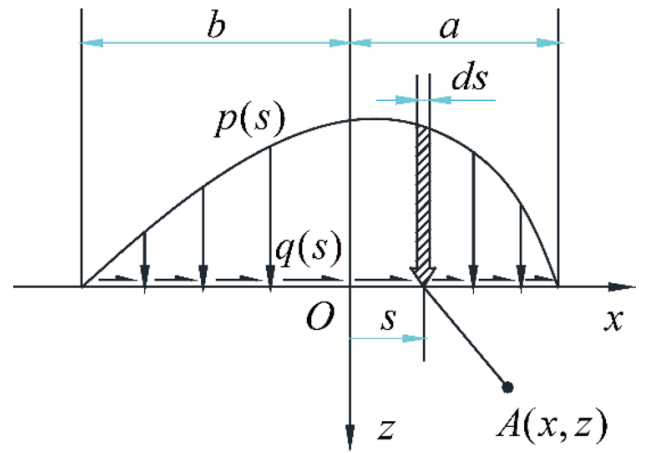

Fig. 2 Elastic half-space under load

On the contact surface, the total stresses on the contact surface must be equal to the applied forces $P$ and $Q$; that is, the relationships are represented as follows:

$P=\int_{\Omega} p(x) d x$

$Q=\int_{\Omega} q(x) d x$

The stress field in the subsurface (see Fig. 2) is determined by the following equation [2]:

$$
\begin{aligned}
& \sigma_{x}=-\frac{2 z}{\pi} \int_{-b}^{a} \frac{p(s)(x-s)^{2} d s}{\left\{(x-s)^{2}+z^{2}\right\}^{2}}-\frac{2}{\pi} \int_{-b}^{a} \frac{q(s)(x-s)^{3} d s}{\left\{(x-s)^{2}+z^{2}\right\}^{2}} \\
& \sigma_{z}=-\frac{2 z^{3}}{\pi} \int_{-b}^{a} \frac{p(s) d s}{\left\{(x-s)^{2}+z^{2}\right\}^{2}}-\frac{2 z^{2}}{\pi} \int_{-b}^{a} \frac{q(s)(x-s) d s}{\left\{(x-s)^{2}+z^{2}\right\}^{2}} \\
& \tau_{x z}=-\frac{2 z^{2}}{\pi} \int_{-b}^{a} \frac{p(s)(x-s) d s}{\left\{(x-s)^{2}+z^{2}\right\}^{2}}-\frac{2 z}{\pi} \int_{-b}^{a} \frac{q(s)(x-s)^{2} d s}{\left\{(x-s)^{2}+z^{2}\right\}^{2}}
\end{aligned}
$$

Using Eq. (5), we can determine the stress components of any point $A(x, z)$ in the subsurface. Conversely, if the stress fields in the subsurface are known, it is possible but challenging to calculate the distribution of loading on the contact region [16]. A combination of photoelasticity experiments and simulations can address this challenge.

\subsection{Contact pressure determination by linear distribution assumption}

Regarding the determination of the contact pressure distribution of the slider-on-block contact, for convenience, the normal pressure was assumed to be a linear distribution [27]. The influence of the friction coefficient on the contact pressure distribution basically depends on the dimensions of the slider and the position of the traction force $Q$; that influence is determined in the following four cases. First, for frictionless conditions (or the $Q$ direction 
as a line in the contact interface), the pressure is evenly distributed as $p(x)=P / l$, where $I$ is the length of the slider. Second, for low friction conditions $(0<\mu \leq l /(6 h)$, where $h$ is the arm of $P$ from the contact surface), the pressure field forms as a trapezoidal distribution. Third, for high friction conditions $(I /(6 h)<\mu \leq I /(2 h))$, the contact pressure field is a triangular distribution. Finally, if $\mu \geq l /(2 h)$, the local contact is almost attributed to the point contact (in 2D), and the motion is unusual. The individual contact pressure equations of each case can be referenced in article [27]. These rules are very helpful in predicting the preliminary friction conditions.

\section{Experimental details}

\subsection{Photoelasticity method}

For direct observation of the plane stress field, the photoelasticity experimental method was employed. This method applies the stress-optical law to determine the stress distribution on a specimen surface or an area of interest, which is based on the special properties of some isotropic transparent materials, such as epoxy resin and polycarbonate. Under polarized light and stressed conditions, it is possible to collect interference patterns that are a response to the principal stress difference (isochromatic) and its direction (isoclinic) in the materials.

A schematic representation of the photoelasticity, which is used to capture the isochromatics, is arrayed in Fig. 3a. A light source, two polarizers, two quarter-wave plates, a contact part and a CCD camera are included. The light source can be used as a monochromatic or white light source. The optical axis of the first polarizer is vertical, and the fast axis of the first quarter-wave plate subtends an angle of $\pi / 4$ with a reference axis ox. The specimen is placed between two quarter-wave plates. The second quarter-wave plate, for which the fast axis subtends an angle of $3 \pi / 4$ with a reference axis $o x$, is placed later. An analyzer with its optical axis of horizontal is then placed. Finally, a CCD camera is placed to capture the photos. With this optical device, it is possible to obtain interference patterns that reflect the principal stress difference, according to the formula below:

$\sigma_{1}-\sigma_{2}=\mathrm{N} \frac{f_{\alpha}}{d}$

where $f_{\alpha}$ is the material stress fringe coefficient, which depends on the birefringent material type and the wavelength of incident light. This parameter can be determined by using pure bending beam experiments [15] or a disk under diametral compression tests [28]; $N$ is the (a)

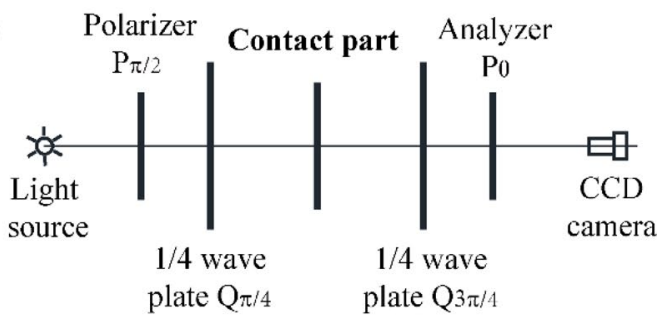

(b)

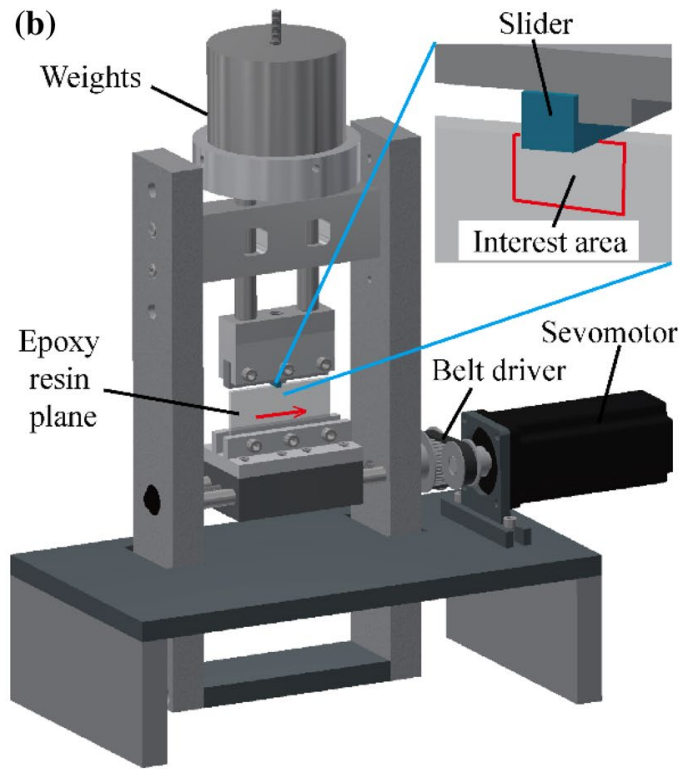

Fig. 3 Photoelasticity experiment a schematic of photoelasticity components, $\mathbf{b}$ experimental apparatus

isochromatic fringe order number, and $d$ is the specimen thickness.

\subsection{Experimental apparatus, materials and tests}

The experimental apparatus was designed as shown in Fig. 3b. For convenient recording, the indenter parts (including the L-shaped body) only moved vertically, and the horizontal movement was restricted, while the sliding parts that the block were fixed on could move along the horizontal direction; thus, the observed contact region was unchanged during the experiments. A servomotor was used to control the velocity of the sliding parts. The dead weights were used to keep a constant load during the test; thus, the weight values could be changed to study the effect of loading.

The transparent block was made by epoxy resin with dimensions of $80 \mathrm{~mm} \times 50 \mathrm{~mm} \times 4 \mathrm{~mm}$ for the width, height, and thickness, respectively. The epoxy resin stress fringe coefficient was determined to be $14.6 \mathrm{kN} /$ (m.fringe) using a four-point bending test [15]. The slider 
Table 1 The properties of the materials

\begin{tabular}{llll}
\hline Material & $\begin{array}{l}\text { Young's } \\
\text { modulus } \\
(\mathrm{GPa})\end{array}$ & Poisson's ratio & Density $\left(\mathrm{kg} / \mathrm{m}^{3}\right)$ \\
\hline Epoxy resin & 2.1 & 0.4 & 1190 \\
Polycarbonate & 1.78 & 0.36 & 1200 \\
Carbon steel & 210 & 0.29 & 7850 \\
\hline
\end{tabular}

was composed of polycarbonate $4 \mathrm{~mm}$ in width, $4 \mathrm{~mm}$ in height and $10 \mathrm{~mm}$ in length. The L-shaped body was made of carbon steel with an overhang of $2 \mathrm{~mm}$. The material properties of Young's modulus, Poisson's ratio and density of all the objects are shown in Table 1.

The average friction coefficient between the epoxy resin (block) and polycarbonate (slider) was measured by this experimental equipment, and its average value equaled 0.35 . Here, Coulomb's formula $\mu=Q / P$ was used to compute the coefficient of friction, where $P$ is the normal force determined by the gravity of the total weight and $Q$ is the friction force, which was measured by employing the HP-1 K Digital Push Pull Force Gauge, determining the pushing force when the sliding parts moved. The coefficient of friction between the carbon steel (L-shaped body) and polycarbonate (slider) was measured using ring-block wear testers with a value of approximately 0.25 [16].

The experimental processes were conducted at room temperature under dry friction conditions. A monochromatic light source with a wavelength of $623 \mathrm{~nm}$ was used in this work. Loads of $83 \mathrm{~N}, 93 \mathrm{~N}, 103 \mathrm{~N}$ and $112 \mathrm{~N}$ were employed in the experiments. In the slip state, the servomotor transmitted a velocity of $0.5 \mathrm{~mm} / \mathrm{s}$ to the sliding parts. The CCD camera was used to capture pictures and record videos during the experiments.

\section{Finite element simulation}

The simulation was performed by the finite element method, which is suitable for the dynamic problems of nonlinear contact, large deformations, high speed dynamics, failure of materials, coupled temperature, etc. The simulation was performed by commercial Abaqus Standard/ Explicit codes with $2 \mathrm{D}$ elastic half space assumptions. The contact configuration model is shown in Fig. 4, and the material properties are inserted according to Table 1, unified with the experiments. The finite element meshing is shown in Fig. 4b, and the mesh is transferred from coarse to fine via edge seedlings. The finest areas are around the contact regions, with a mesh size of $0.01 \mathrm{~mm}$. This meshing method leads to better contact stress results with a reasonable computation analysis time [23]. For the $2 \mathrm{D}$
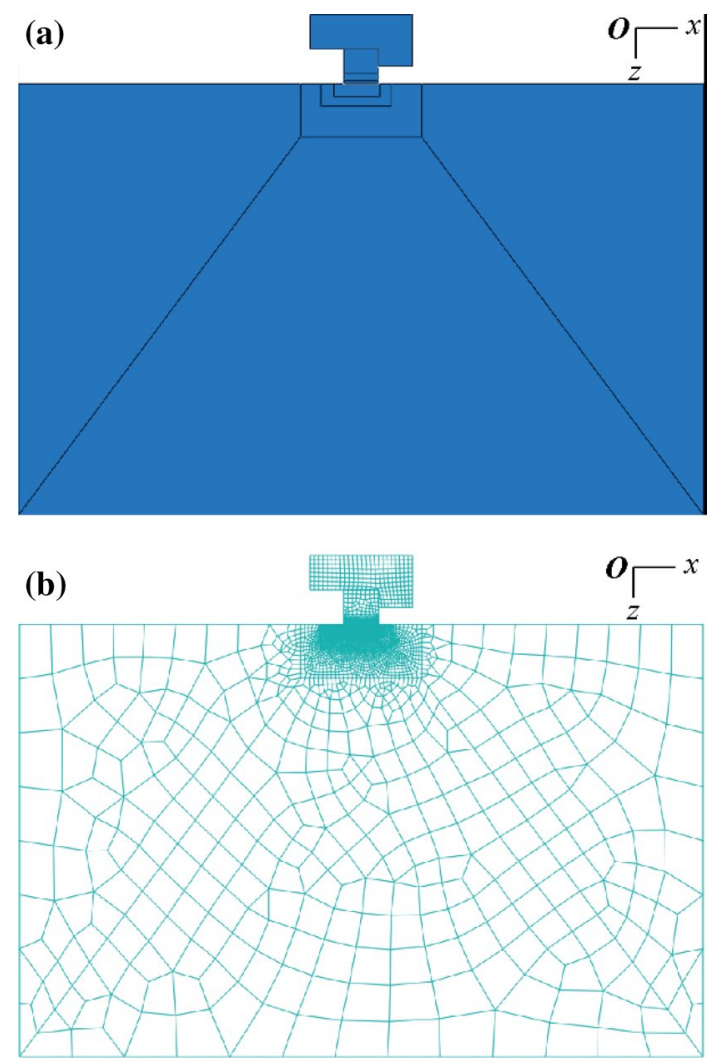

Fig. 4 2D simulation model a dividing the parts to optimize the grid segments, $\mathbf{b}$ meshing

dynamic explicit problem, the explicit linear quadrilateral and plane strain element is used to obtain better results, so this element is embedded in each part of the model. Surface-to-surface contact is employed for the slider-block and slider-L-shaped body on their contact areas. Friction behavior is employed in the interaction using the penalty contact algorithm. The coefficients of the two couples of friction pairs are the same as those in the experiments: 0.25 for the carbon steel-polycarbonate and 0.35 for the epoxy-polycarbonate.

The time step is assigned within the time period of $0.3 \mathrm{~s}$, in which a scale to target time increment of $1.10^{-6} \mathrm{~s}$ is created in semiautomatic mass scaling with the purpose of analyzing the model over its natural time period [23]. The L-shaped body with a long edge of $12 \mathrm{~mm}$ is restricted from motion in the $x$ direction by the left and right edges of the body, and a pressure $p_{L}$ acts on the top of the body (as shown in Fig. 1b), corresponding to the loads in the experiment. $p_{L}$ is calculated as shown in Table 2. The bottom edge of the block is restricted from motion in the vertical direction, and the left and right edges of the block are moved at a speed of $0.5 \mathrm{~mm} / \mathrm{s}$ from left to right in the slip state. To minimize stress wave propagation in the simulation, the velocity of the block and the pressure are ramped 
Table 2 Pressures in the simulation corresponding to the loads in the experiments

\begin{tabular}{ll}
\hline Load $(\mathrm{N})$ & $p_{L}(\mathrm{MPa})$ \\
\hline 83 & 1.73 \\
93 & 1.93 \\
103 & 2.13 \\
112 & 2.34 \\
\hline
\end{tabular}

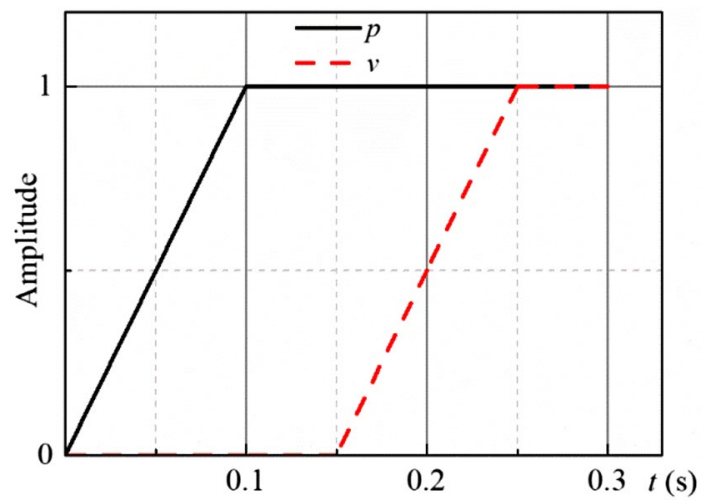

Fig. 5 Amplitudes of velocity $v$ and pressure $p$ up gradually from zero with a smooth step amplitude, as shown in Fig. 5.

\section{Results and discussion}

Use of the photoelasticity experiment can directly determine the principal stress difference field, so comparison of the experimental results with the finite element simulation results can help to assess the accuracy of the simulation. Moreover, we utilize simulation data to show some other results that cannot be determined by experiments, such as the contact pressure and frictional stress. In this section, the results for the principal stress difference fields in the subsurface and the contact pressure and frictional stress distributions at the contact region for the static and slip states are given and discussed.

\subsection{Static contact}

\subsubsection{Principal stress difference}

For the case of static contact, the block was fixed, and the load was changed by replacing the weights and total loads as $83 \mathrm{~N}, 93 \mathrm{~N}, 103 \mathrm{~N}$ and $112 \mathrm{~N}$. The principal stress difference (Tresca stress) fields in the block were captured in both the experiments and simulations and then compared in Figs. 6 and 7. Using Eq. (6), we can determine the fringe order $N=1$ in the photoelasticity figures to obtain
Fig. 6 Experimental results for the principal stress difference field under different loads for static contact
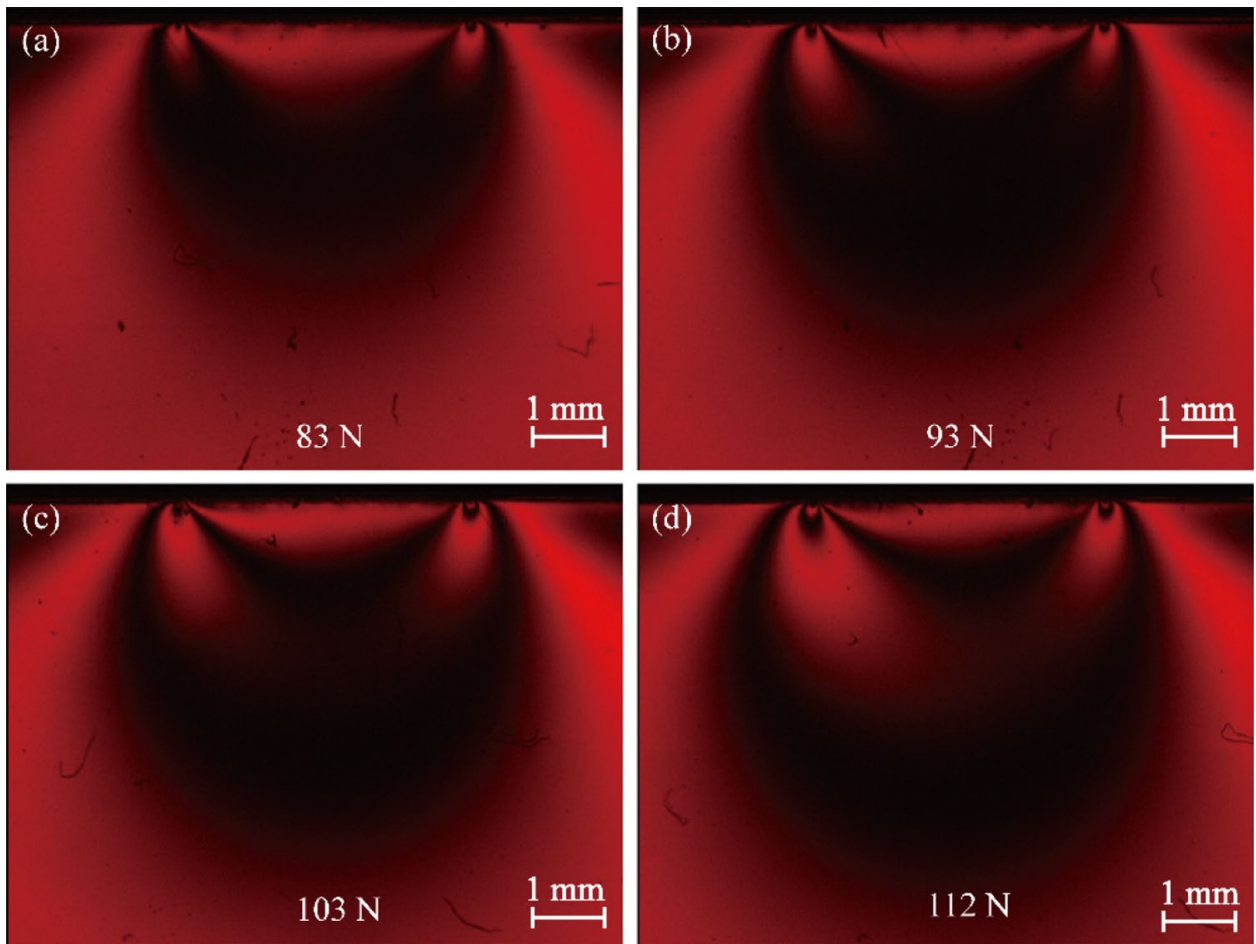
Fig. 7 Simulation results for the principal stress difference fields under different loads for static contact
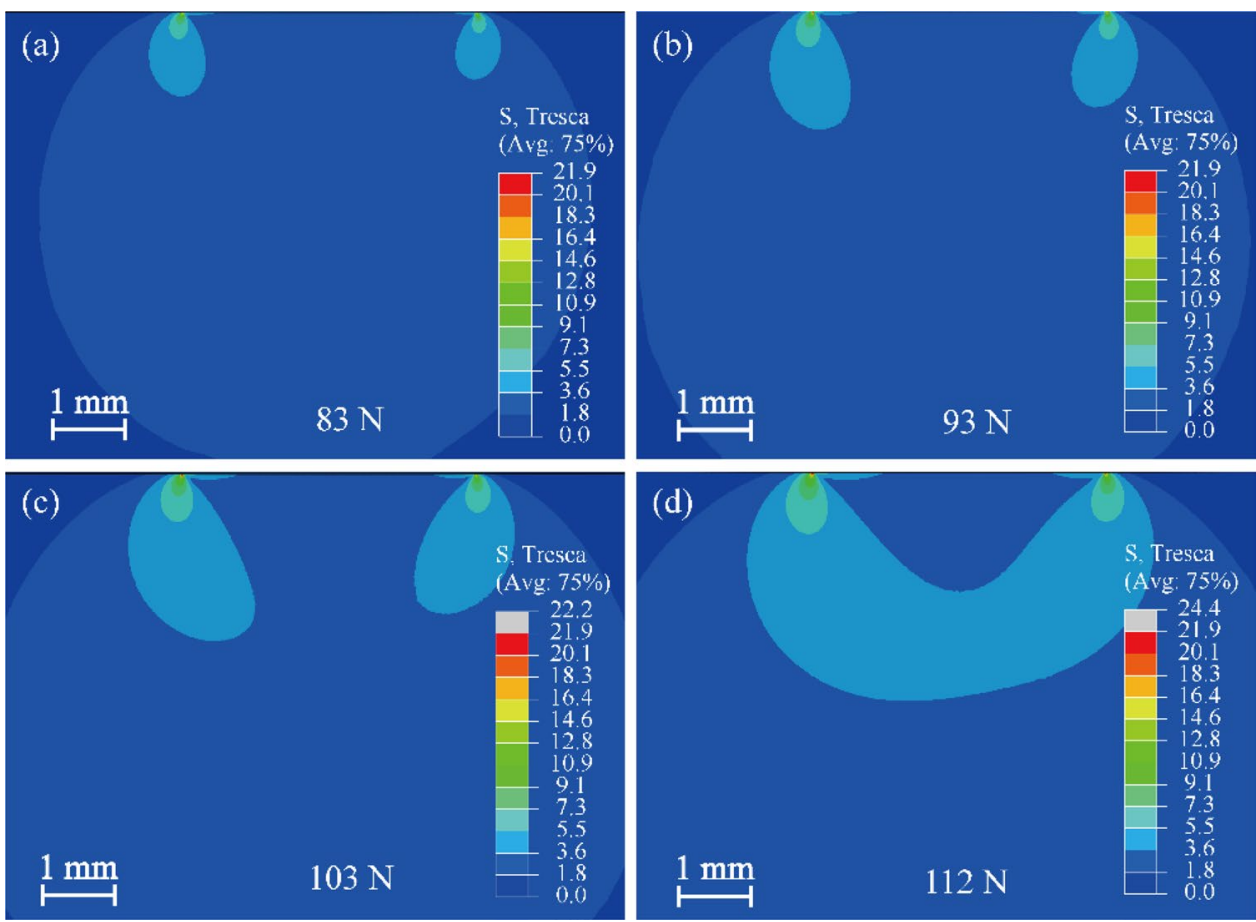

the principal stress difference $\left(\sigma_{1}-\sigma_{2}\right)$, which equals 3.6 MPa (Fig. 6); compared to the simulation figures, there is a coincidence between the experimental and simulation results. Basically, the principal stress difference had a symmetrical distribution with the largest value mainly appearing in the local areas below the two edges of the slider. However, all the stress fields (including the contact pressure and frictional stress fields mentioned below) were slightly deflected to the left because of the L-shaped body pushing the slider to the left. With increasing load, the maximum principal stress difference values significantly increased. This change is expressed by a larger number of isochromatic fringe orders (in the experiments) and the increasing maximum value of the principal stress difference (in the simulations).

Figure 8 shows a comparison of the principal stress differences of section $A-A, z=0.18 \mathrm{~mm}$ from the contact region, under a load of $112 \mathrm{~N}$ from simulation and experiment. Basically, the two curves follow the same trends. This result shows the coincidence of the simulation and experimental results.

\subsubsection{Contact pressure}

Based on the simulation, the distributions of pressure on the contact surface were determined for the static state, shown in Fig. 9. Due to the symmetry of the model, the pressure had a symmetrical distribution. When the load increased, the contact pressure tended to increase (see

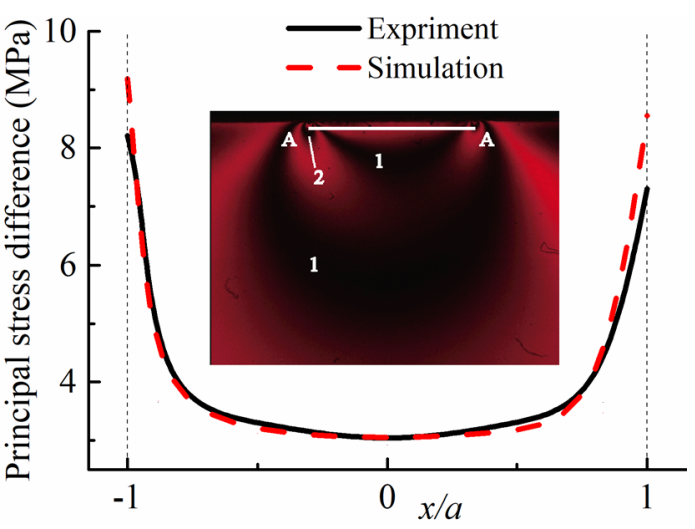

Fig. 8 Comparison of principal stress differences in section A-A (at $z=0.18 \mathrm{~mm}$ )

Fig. 9a). Figure 9b shows a comparison between the results of the simulation, elastic theory and the assumption of a linear distribution under a load of $112 \mathrm{~N}$. It should be noted that for the elastic theory, the contact pressure was distributed as $p(x)=P /\left(\pi \sqrt{a^{2}-x^{2}}\right)$ for a rigid flat punch contact with an elastic half space [2], shown by bluedotted line in Fig. 9b. There was a difference between the simulation curve and the theoretical curve. In the middle of the contact region $(x / a \sim 0)$, the simulation value was much higher than the theoretical value; however, far from this area $(x / a \rightarrow \pm 1)$, the theoretical curve slowly increased and then crossed the simulation curve at a special value of the contact pressure, shown Fig. 9b. When $x /$ a reached \pm 1 , 

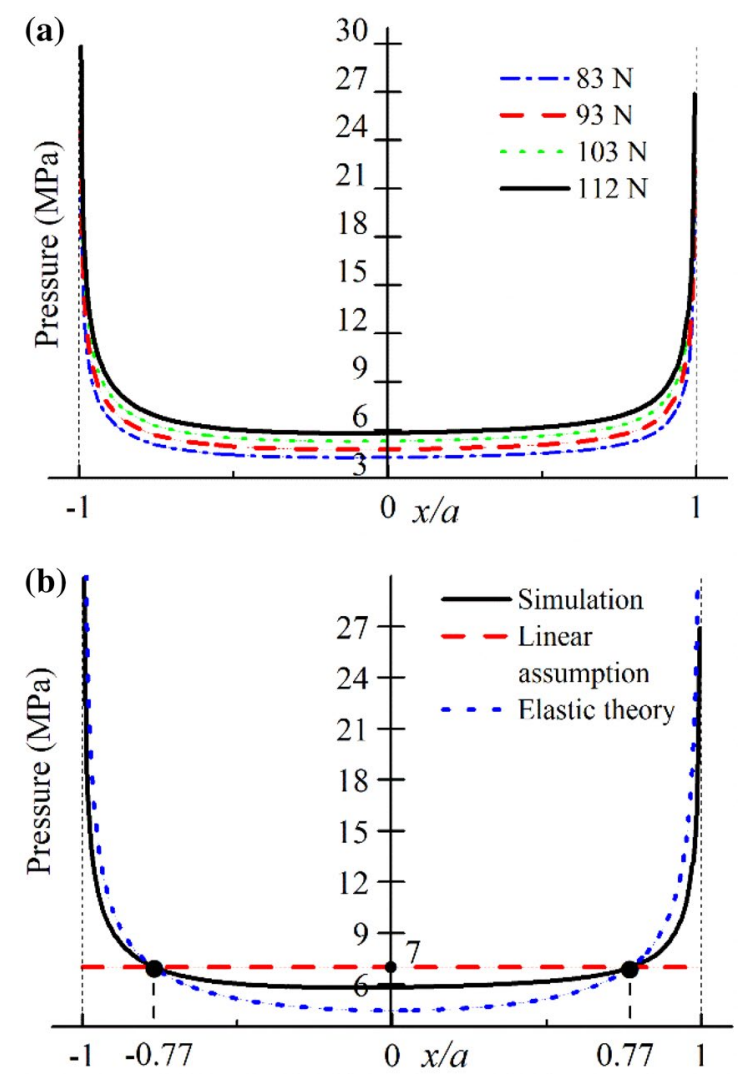

Fig. 9 Contact pressure distribution at static state a different loads, b comparison under load of $112 \mathrm{~N}$

the theoretical pressure was infinite, but the simulation results gave the limit values. For the linear assumption, the pressure distribution was evenly distributed as a horizontal line. From Fig. 9b, we can see that all three curves intersected at the average contact pressure of $7 \mathrm{MPa}$, where $x / a= \pm 0.77$. When changing the load, the average pressure value changed accordingly, but the simulation, elastic theory and linear assumption curves also intersected at $x / a= \pm 0.77$ (see Table 3).

Figure 10 shows the effect of the friction coefficient on the pressure distribution for the static state, with a load of $112 \mathrm{~N}$. It can be seen that in the middle of the contact region $|x / a|<1$, the influence of the friction coefficient on the pressure distribution was vague, but near the edges,

Table 3 Intersection of three curves when the load changes

\begin{tabular}{llll}
\hline Load $(\mathrm{N})$ & Intersected & Position & \\
\cline { 3 - 4 } & & Pressure (MPa) & $x / a$ \\
\hline 83 & Yes & 5.2 & \pm 0.77 \\
93 & Yes & 5.8 & \pm 0.77 \\
103 & Yes & 6.4 & \pm 0.77 \\
112 & Yes & 7.0 & \pm 0.77 \\
\hline
\end{tabular}

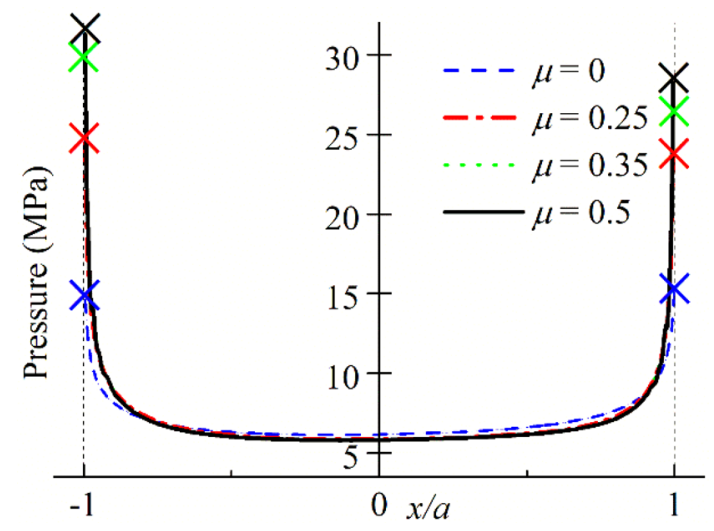

Fig. 10 Effect of the friction coefficient on the pressure distribution in the static state

the maximum values of the contact pressure (denoted $x$ ) increased as the friction coefficient increased. With this result, it can be derived that the contact pressure reached an average value at $x / a= \pm 0.77$ and that there was no dependence on the friction coefficient.

\subsubsection{Frictional stress}

In the simulation, the shear stress on the contact surface represented the frictional stress. Accordingly, for static contact, there were two frictional stress peaks at the two sides (with opposite values); however, in the middle of the contact region, the frictional stress was small and reached zero in the middle, shown in Fig. 11. This result was consistent with the observations of the fretting fatigue phenomenon of a sphere-on-flat surface, and accordingly, the risk of fatigue failure was greatest at the edges [2].

As the load and friction coefficient increased, the value of the stress peak increased accordingly (see Fig. 11).

\subsection{Sliding contact}

\subsubsection{Principal stress difference}

For the sliding case, the block was moved from left to right at a velocity of $0.5 \mathrm{~mm} / \mathrm{s}$. Similar conditions of loads and sliding speed were applied in both experiments and simulations. The isochromatic patterns in the block were captured in the experiments, and the principal stress difference fields in the simulations were taken, as shown in Fig. 12 and Fig. 13, respectively. Here, the stress was mainly concentrated in the local area beneath the left edge of the slider, which was the front edge in the moving direction. The line contact became a point contact (in 2D). For the process of sliding over time, the contact friction often occurred as vibrations (the reader can view the attached 
(a)

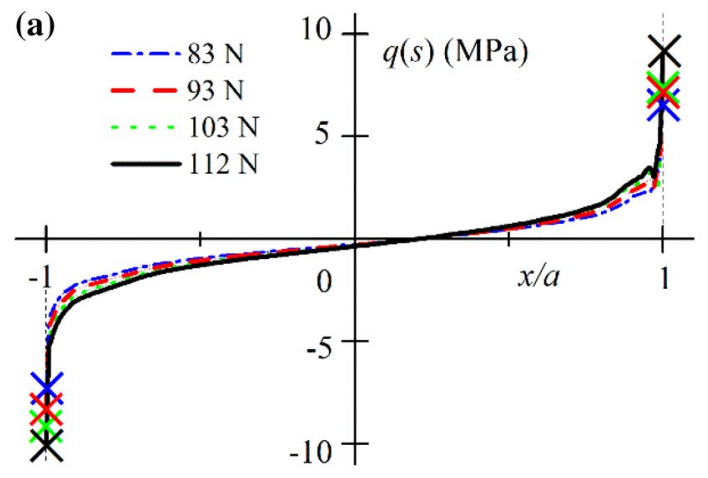

(b)

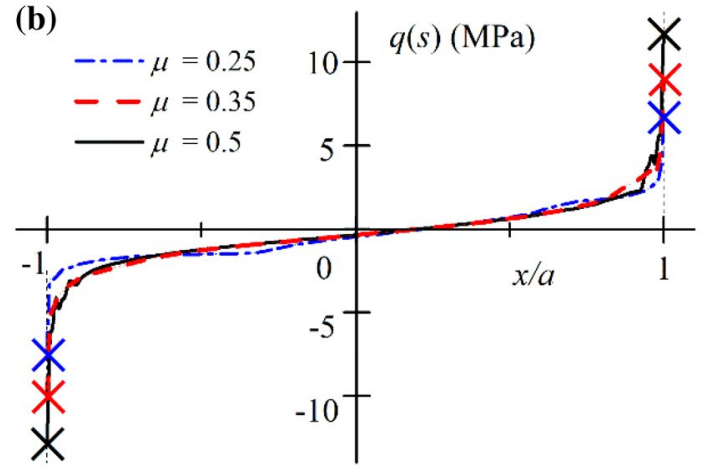

Fig. 11 Frictional stress distribution at static contact a load change, b friction coefficient change, load of $112 \mathrm{~N}$ video). Therefore, the results of the isochromatic fringes were often interrupted [3], but generally, the stress field tended to focus on the front edge in the moving direction.

Figure 14 shows the results of the principal stress difference extracted from straight line B-B in the block, under a contact surface of $z=0.18 \mathrm{~mm}$ and load of $112 \mathrm{~N}$. We can see that the experiment and simulation curves basically had the same rules. However, the stress wave propagation during the moving process was unavoidable, leading to error between the experiment and simulation results.

\subsubsection{Contact pressure}

Figure 15 shows the contact pressure distribution along the contact surface of the block. The symmetry of the pressure distribution for static contact was broken. This maximum pressure appeared on the left and decreased toward the right. Figure $15 \mathrm{~b}$ shows a comparison between the simulation result and the linear assumption result for a load of $112 \mathrm{~N}$ and a slider-block friction coefficient of 0.35 . The distribution of pressure under the linear assumption was expressed as $p(x)=6.89(1.15-x)$ MPa when $-2 \mathrm{~mm} \leq x<1.15 \mathrm{~mm}$ and $p(x)=0$ when $1.15 \mathrm{~mm}<x \leq 2 \mathrm{~mm}$ (see Fig. $1 \mathrm{~b}$, with $a=2 \mathrm{~mm}$ ) by applying the formulas of article [27]. Here, we can see that the linear assumption distribution result and simulation result were different. When $1.15 \mathrm{~mm}<x \leq 2 \mathrm{~mm}$ or $0.58<\mathrm{x} / \mathrm{a} \leq 1$, $p(x)$ equaled zero for the linear assumption but did not reach zero in the simulation. When $-2 \mathrm{~mm} \leq x<1.15 \mathrm{~mm}$, in the case of the linear assumption, $p(x)$ linearly increased
Fig. 12 Experimental results of the principal stress difference fields under different loads in the sliding state
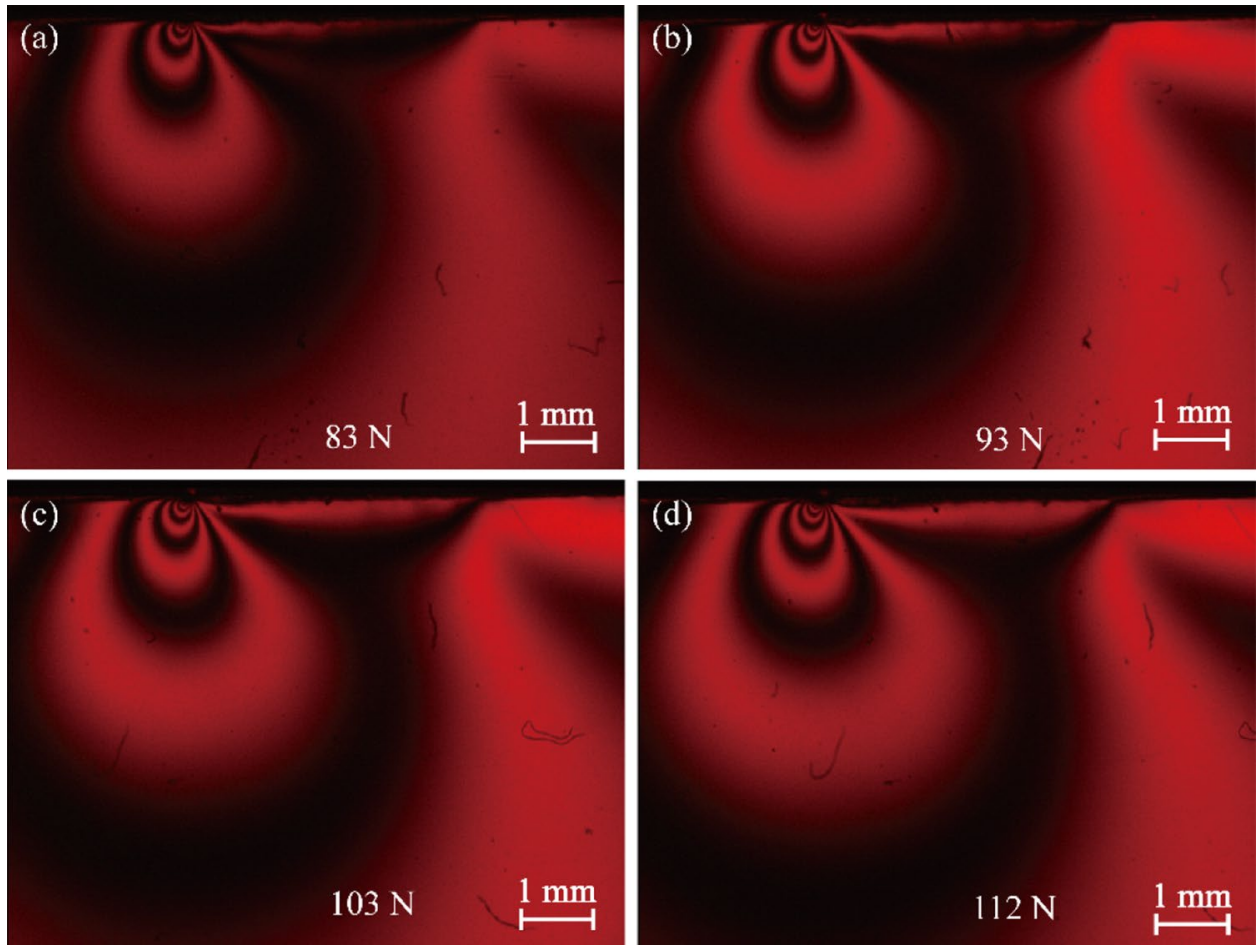
Fig. 13 Simulation results of the principal stress differences under different loads in the sliding state
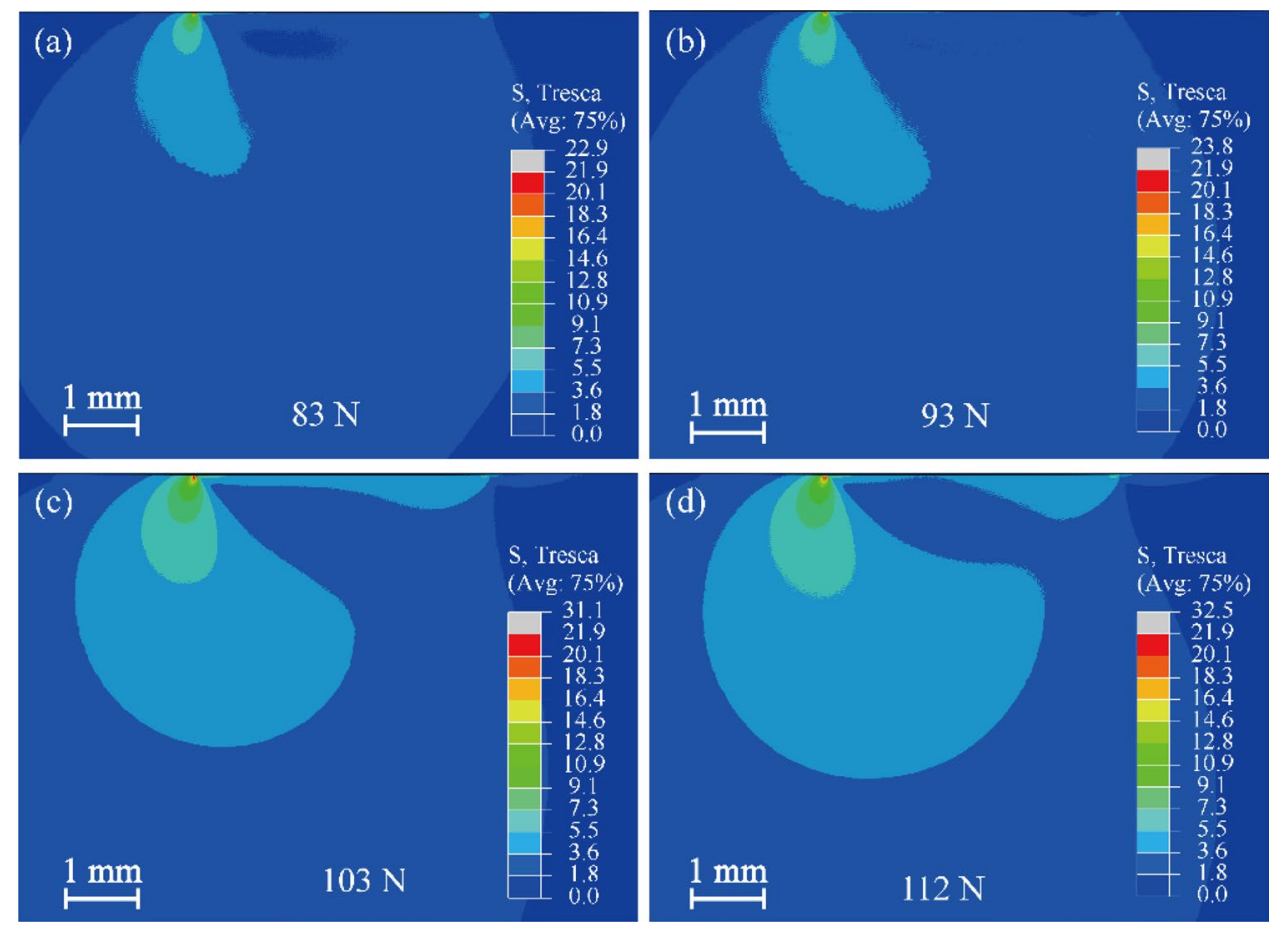

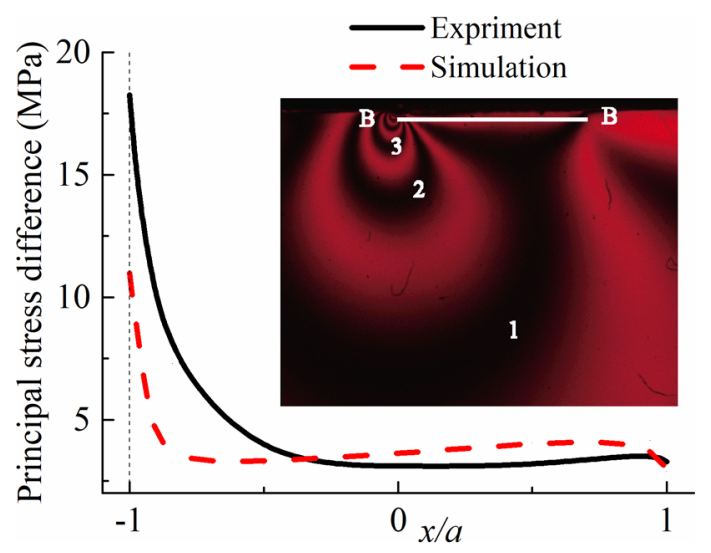

Fig. 14 Comparison of principal stress differences in section B-B (at $z=0.18 \mathrm{~mm}$ )

and reached a maximum value of $21.7 \mathrm{MPa}$ but gradually increased and reached a maximum value of $39.1 \mathrm{MPa}$ in the simulation. Moreover, in both simulation and test processes, the contact sometimes discontinued on the right side because of the oscillation phenomenon caused by intermittent contact.

Figure 16 shows the pressure distributions on the contact surface for different friction coefficients when the block and slider moved relative to each other. Here, the friction coefficients of the slider block were $0,0.25,0.35$ and 0.5 . From the figure, for the frictionless case, the pressure field was the same as the static contact distribution; see the blue-dashed line in Fig. 16. However, when the
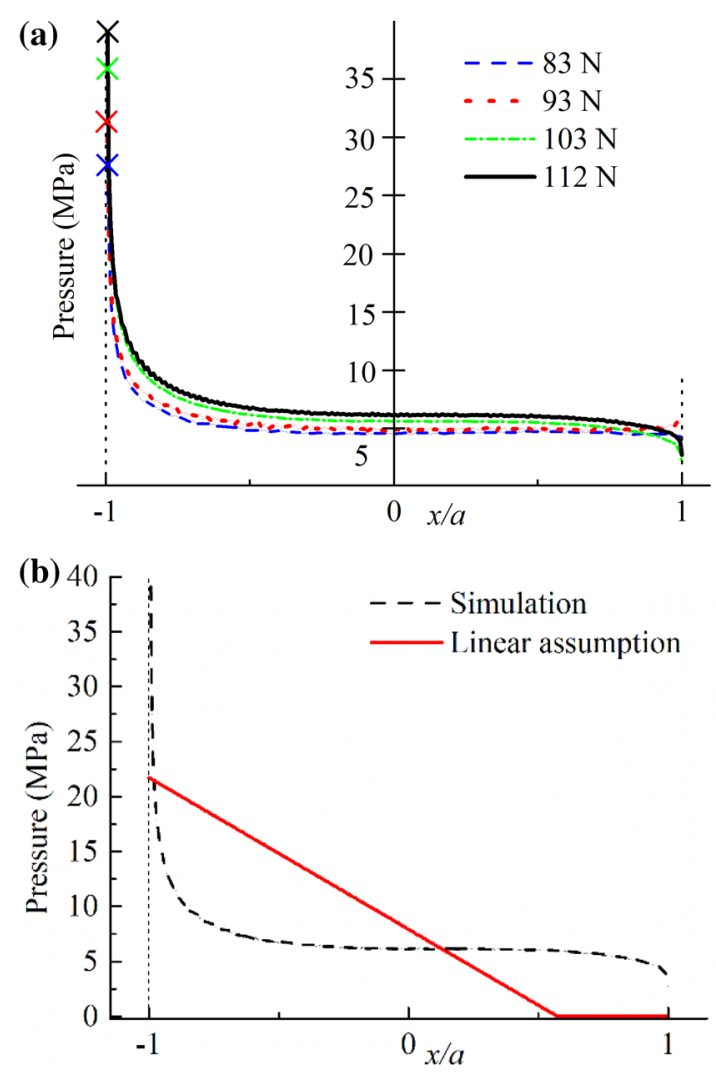

Fig. 15 Pressure distribution at slip state a load variation, b comparison of simulation and linear distribution assumption, friction coefficient of 0.35 and load of $112 \mathrm{~N}$ 


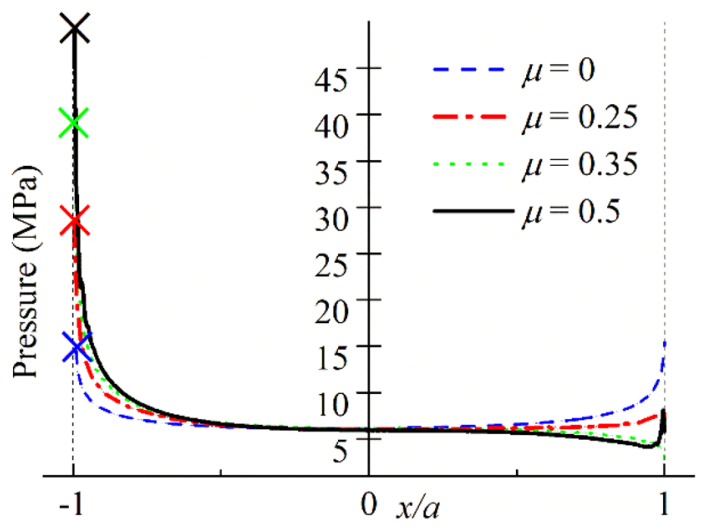

Fig. 16 Pressures for different friction coefficients at slip state

coefficient of friction increased, the pressure distribution gradually deflected toward the moving direction, leading to a gradually higher left pressure peak but a gradually lower right pressure peak that may reach zero. Furthermore, because the sliding friction process was a jerking motion [3], the pressure on the right side fluctuated and sometimes reached zero (noncontact).

\subsubsection{Frictional stress}

Figure 17 shows the results for the frictional stress on the contact surface for the slip state. We can see that, in contrast with the static contact, there were no longer two stress peaks at two sides but only one peak on the left side, which was also observed in the contact pressure distribution. The frictional stress reached zero in the middle region for the static state and moved to the right edge for the slip state. Compared with the sphere-on-flat contact model, due to the different physical models, the frictional stress of the sphere-on-flat model still exhibited two stress peaks, with the left peak having a higher value than the right [4].

As the load and friction coefficient increased, the value of the frictional stress tended to increase accordingly (see Fig. 17). This trend is consistent with the theory of contact.

\section{Conclusions}

Photoelasticity experiments combined with finite element simulation were applied to investigate the contact stress field of slider-on-block contact for different states. Interference patterns were obtained by the photoelasticity method under different loads. Finite element simulations
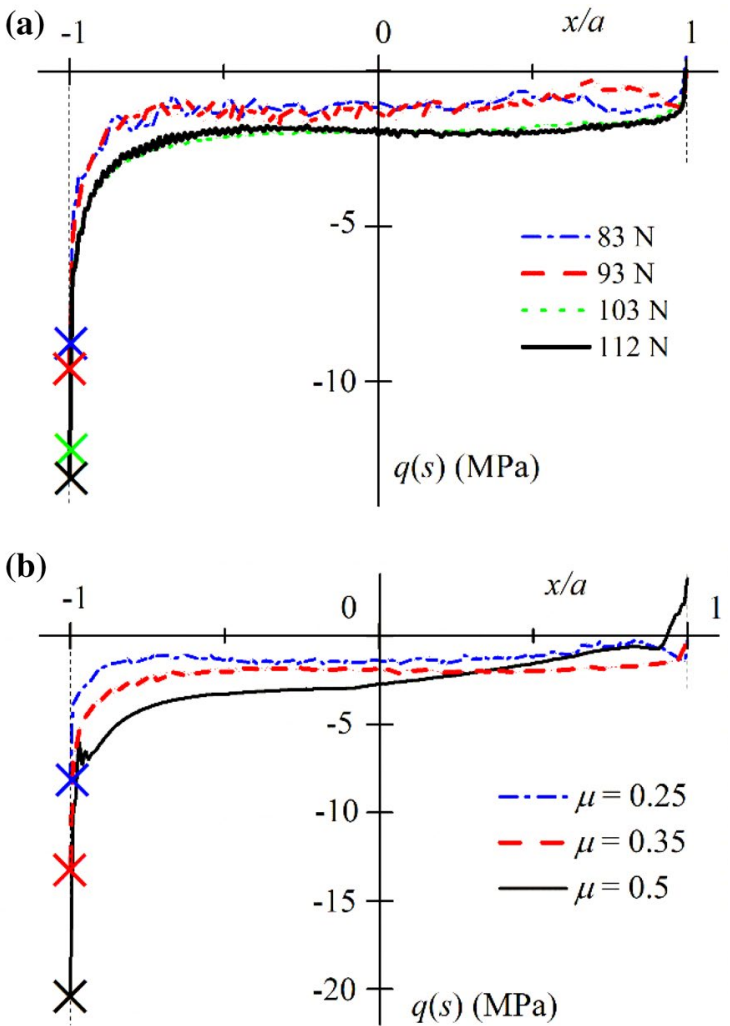

Fig. 17 Frictional stress distributions for slip state a) Load variation, b friction coefficient variation and load of $112 \mathrm{~N}$

were conducted under the same conditions as in the experiments. Some of the main conclusions are as follows:

1. The photoelasticity method was employed to directly observe the principal stress difference fields of the slider-on-block contact model for static and slip states.

2. The images of the principal stress difference field obtained from the simulation were in accordance with the experimental results, thus allowing the utilization of simulation data to fully display the parameters of the contact stress field, such as the pressure and frictional stress on the contact surface.

3. The results show that the contact stresses concentrated at the edges of the slider for the static state but deflected completely to the front edge of the moving direction for the sliding state. These results lead us to conclude that the most likely area of failure is the front edge of the moving direction.

4. The effects of loads and friction coefficients on the contact stress distributions were significant. However, the variation of these factors did not change the position of the contact pressure average $(x / a= \pm 0.77)$ in the static state. 
Acknowledgements The authors would like to acknowledge the financial support of the National Natural Science Foundation of China (Grant No. 51575190)

\section{Compliance with ethical standards}

Conflict of interest The authors declare that they have no competing interests

\section{References}

1. Wen SZ, Huang P (2011) Interface science and technology. Tsinghua University Press, Beijing

2. Johnson KL (1985) Contact mechanics. Cambridge University Press, Cambridge

3. Wen SZ, Huang P (2012) Principles of tribology. Tsinghua University Press, Beijing

4. Hamilton GM, Goodman LE (1966) The stress field created by a circular sliding contact. J Appl Mech 33(2):371-376. https://doi. org/10.1115/1.3625051

5. Keer LM, Mowry DB (1978) The stress feld created by a circular sliding contact on transversely isotropic spheres. Int J Solids Struct 15(1):33-39. https://doi.org/10.1016/0020-7683(79)90041 $-6$

6. Johnson K (1982) One hundred years of Hertz contact. Proc Inst Mech Eng 196(1):363-378. https://doi.org/10.1243/ PIME_PROC_1982_196_039_02

7. Popov VL (2010) Contact mechanics and friction. Springer, Berlin

8. Komarnicki P, Stopa R, Kuta $\measuredangle$, Szyjewicz D (2017) Determination of apple bruise resistance based on the surface pressure and contact area measurements under impact loads. Comput Electron Agric 142:155-164

9. Fukubayashi T, Kurosawa $\mathrm{H}$ (1980) The contact area and pressure distribution pattern of the knee: a study of normal and osteoarthrotic knee joints. Acta Orthop Scand 51(1-6):871-879

10. Lekue J, Dörner F, Schindler C (2017) On the source of the systematic error of the pressure measurement film applied to wheel-rail normal contact measurements. J Tribol 140(2):024501-024504. https://doi.org/10.1115/1.4037358

11. Brewster $D$ (1816) On the communication of the structure of doubly-refracting crystals to glass, murite of soda, flour spar, and other substances by mechanical compression and dilation. Philos Trans R Soc Lond 106:156-178. https://doi.org/10.1098/ rstl.1816.0011

12. Brewster $D$ (1815) Experiments on the depolarisation of light as exhibited by various mineral, animal, and vegetable bodies, with a reference of the phenomena to the general principles of polarisation. Philos Trans R Soc Lond 105(1):29-53. https://doi. org/10.1109/ICCAS.2014.6987986

13. Dally JW, Chen YM (1991) A photoelastic study of friction at multipoint contacts. Exp Mech 31(2):144-149. https://doi. org/10.1007/BF02327567

14. Hariprasad MP, Ramesh K, Prabhune BC (2018) Evaluation of conformal and non-conformal contact parameters using digital photoelasticity. Exp Mech 58(8):1249-1263. https://doi. org/10.1007/s11340-018-0411-6

15. Fang $Y$, He J, Huang $P$ (2017) Experimental and numerical analysis of soft elastohydrodynamic lubrication in line contact. Tribol Lett 65(2):42. https://doi.org/10.1007/s11249-017-0825-9

16. Zhan W, Fang Y, Hoang VC, Huang P (2018) Instantaneous plane stress observation and numerical simulation during wear in an initial line contact. Tribol Lett 66(3):90. https://doi.org/10.1007/ s11249-018-1042-x

17. Ajovalasit A, Petrucci G, Scafidi M (2015) Review of RGB photoelasticity. Opt Lasers Eng 68:58-73. https://doi.org/10.1016/j. optlaseng.2014.12.008

18. Ajovalasit A, Pitarresi G, Zuccarello B (2007) Limitation of carrier fringe methods in digital photoelasticity. Opt Lasers Eng 45(5):631-636. https://doi.org/10.1016/j.optlaseng.2006.08.008

19. Zuccarello B, Tripoli G (2002) Photoelastic stress pattern analysis using Fourier transform with carrier fringes: influence of quarter-wave plate error. Opt Lasers Eng 37(4):401-416. https://doi. org/10.1016/s0143-8166(01)00103-8

20. Hariprasad MP, Ramesh K (2018) Analysis of contact zones from whole field isochromatics using reflection photoelasticity. Opt Lasers Eng 105:86-92. https://doi.org/10.1016/j.optla seng.2018.01.005

21. Briñez JC, Restrepo Martínez A, Branch JW (2018) Computational hybrid phase shifting technique applied to digital photoelasticity. Optik 157:287-297. https://doi.org/10.1016/j.ijleo .2017.11.060

22. Ramakrishnan V, Ramesh K (2017) Scanning schemes in white light photoelasticity-Part II: Novel fringe resolution guided scanning scheme. Opt Lasers Eng 92:141-149. https://doi. org/10.1016/j.optlaseng.2016.05.010

23. Siswanto WA, Muniandy N, Mohd Tobi AL, Tamin M (2015) Contact pressure prediction comparison using implicit and explicit finite element methods and the validation with Hertzian theory. Int J Mech Mechatron Eng 15:1-8

24. Bhushan B, Peng W (2001) Contact mechanics of multilayered rough surfaces. Appl Mech Rev 55(5):435-480. https://doi. org/10.1115/1.1488931

25. Bhushan B (1998) Contact mechanics of rough surfaces in tribology: multiple asperity contact. Tribol Lett 4(1):1-35. https://doi. org/10.1023/a:1019186601445

26. Masoudi Nejad R, Farhangdoost K, Shariati M (2015) Numerical study on fatigue crack growth in railway wheels under the influence of residual stresses. Eng Fail Anal 52:75-89. https:// doi.org/10.1016/j.engfailanal.2015.03.002

27. Huang P, Yang Q (2014) Theory and contents of frictional mechanics. Friction 2(1):27-39. https://doi.org/10.1007/s4054 4-013-0034-y

28. Lei ZK, Yun H, Zhao YR, Pan XM (2009) Automatic determination of fringe constant in digital photoelasticity. Eng Mech 26(12):40-443

Publisher's Note Springer Nature remains neutral with regard to jurisdictional claims in published maps and institutional affiliations. 